\title{
ON THE PROBLEM OF TASK PLANNING IN MULTI-ROBOT SYSTEMS
}

\author{
Zhi Yan, Nicolas Jouandeau and Arab Ali Cherif \\ Advanced Computing Laboratory of Saint-Denis (LIASD) \\ Paris 8 University \\ 2 rue de la Liberté, 93526 Saint-Denis, France \\ $\{y z, n, a a\} @ a i . u n i v-p a r i s 8 . f r$
}

\begin{abstract}
Keywords: Task planning, multi-robot systems, coordination.
Abstract: $\quad$ Multi-robot task planning (MRTP) is one of the fundamental problems for multi-robot systems. An important question facing this research topic is, which robot should execute which task so as the expected overall system performance can be maximized? Many approaches have been proposed for such a purpose. This paper investigates the existing works in the field. The approaches have been surveyed and some representatives are compared with detailed results. A brief discussion and further research perspectives are also given at the end of the paper.
\end{abstract}

\section{INTRODUCTION}

The task of coming up with a sequence of actions that will achieve a goal is called planning (Russell and Norvig, 2002). In multi-robot systems (MRS), planning can be used to coordinate robots to accomplish the team mission. Unfortunately, optimal planning for MRS is typically an $\mathcal{N} \mathcal{P}$-hard problem. The challenge then is to have tractable planning that produces good solutions (Dias et al., 2005). Multi-robot planning is usually divided into two aspects: task planning and motion planning. Task planning involves task decomposition and task allocation. So far, research on task planning of MRS is concentrated on task allocation problem, and relatively little on task decomposition problem. In fact, the task decomposition is an important research topic because the effect of task allocation could be directly influenced by it.

\section{MULTI-ROBOT TASK DECOMPOSITION}

The problem of multi-robot task decomposition (MRTD) mainly refers to that the total mission to be completed is decomposed into several single subtasks which can be completed independently by a robot separately, according to the characteristic, requirement and resource allocation of the total mis- sion itself (Chen et al., 2010). (Stone and Veloso, 1999) achieved collaboration between agents through the introduction of formations which decomposes the task space defining a set of roles with associated behaviors. (Botelho and Alami, 1999) presented a decentralized system to describe and perform task planning, decomposition and allocation in multi-robot environments, called $\mathrm{M}+$ protocol. (Zlot and Stentz, 2005) focused on complex tasks that can be decomposed into multiple inter-related subtasks. They addressed the task decomposition problem by generalizing tasks to task trees within a peer-to-peer trading market. (Tang and Parker, 2005) considered that, in typical approaches to multi-robot team working, the decomposition of the team task into subtasks is defined by the human designer in advance of the robot team performance, and this pattern also outlines the available multi-robot task solutions in advance of the mission. So they described a methodology for automatically synthesizing task solutions for heterogeneous multi-robot teams.

\section{MULTI-ROBOT TASK ALLOCATION}

The problem of multi-robot task allocation (MRTA) can be considered as an instance of the well-known optimal assignment problem (OAP). In domains 
where the group dynamics have a significant effect on group performance, MRTA is known to be $\mathcal{N}(\mathcal{P}$ hard (Gerkey and Matarić, 2004) (Dahl et al., 2009). This is the most common case in MRS. The task allocation in MRS is generally undertaken to assign robots to tasks (or tasks to robots) so as to maximize the expected overall system performance. However, because MRTA becomes a dynamic decision problem that varies in time with environmental changes, the static assignment method is no longer applicable. Thus an alternative solution is to iteratively solve the static assignment problem over time.

(Gerkey and Matarić, 2004) gave a formal analysis and domain-independent taxonomy of MRTA problems, in which the MRTA problems have been classified into seven categories according to ability of robot to perform tasks, number of robots required for a task, and manner of the task assignment. They also analyzed and compared some iterated assignment architectures: ALLIANCE (Parker, 1994a), BLE (Werger and Matarić, 2000), and M+ (Botelho and Alami, 1999), and some online assignment architectures: MURDOCH (auction-based) (Gerkey and Matarić, 2002), first-price auctions (market-based) (Stentz and Dias, 1999), and dynamic role assignment (Chaimowicz et al., 2002), for MRTA, respectively.

The contract net protocol (CNP) has been developed by (Smith, 1980), which aims to achieve task assignment with distributed control by a negotiation process in multi-agent systems. So far, most methods of MRTA are based on the CNP model. (Botelho and Alami, 1999) presented M+ system, a scheme for multi-robot cooperation through negotiated task allocation and achievement, which is the first CNP-based approach to MRTA. (Stentz and Dias, 1999) presented the ideas of free market architecture for coordinating a group of robots to achieve a given objective (marketbased approach). This architecture defines revenue and cost functions across the possible plans for executing a specified task. The task is accomplished by dividing it into subtasks and allowing the robots to bid and negotiate to carry out these subtasks. The objective is achieved by individual robots cooperating and competing with each other to further their own self-interests. (Zlot et al., 2002) applied these ideas to multi-robot mapping and exploration problem. This work borrows the market architecture which seeks to maximize benefit while minimizing cost, thus aiming to maximize utility. The benefit is information gained by visiting a goal point, the cost is the estimated distance traveled to reach the goal (by using $\mathrm{D}^{*}$ algorithm (Stentz, 1994)), then the utility is the difference between the benefit and the cost. The market-based coordination architecture has been applied to a Mars exploration scenario (combined with $\mathrm{D}^{*}$ algorithm for robot motion planning). (Gerkey and Matarić, 2002) presented the first online assignment architecture MURDOCH, which uses a first-price auction to assign each task (auction-based approach). The auction proceeds in five steps: task announcement, metric evaluation, bid submission, close of auction, and progress monitoring/contract renewal. The MURDOCH system has been tested in two different domains: a long-term loosely coupled task domain and a short-term tightly coupled box-pushing task. The major differences between auction-based approach and market-based approach are that, 1) auction-based approach uses the bid based on the estimated cost, but market-based approach takes into account both cost and benefit, 2) auction-based approach does not allow task reassignment, but market-based approach allows later reassignment.

In addition, (Dahl et al., 2009) presented an algorithm for task allocation in groups of homogeneous robots, which is based on vacancy chains, a resource distribution strategy common in human and animal societies. This algorithm uses local task selection, reinforcement learning for estimation of task utility, and reward structures based on the vacancy chain framework. (Hanna, 2005) proposed an approach which allows robots to take into account the uncertainty of task execution. They decomposed the MRTA problem into two stages. In the first stage, each robot selects its own tasks based on the expected benefit using Markov decision process (MDP). In the second stage, an auction-based mechanism is applied to assign tasks to robots. (Michael et al., 2008) proposed a distributed market-based coordination algorithm where agents are able to bid for task assignment with the assumption that agents have knowledge of all tasks as well as the maximum number of agents that can be assigned to every individual task. Each auction is performed among neighboring groups of agents and requires only local communication. They verified their algorithm in multi-robot formation control problem. (Shiroma and Campos, 2009) proposed a framework called CoMutaR, which is designed to both tackle task allocation and coordination problems in MRS. This framework enables the single robot to perform multiple tasks concurrently by periodically checking and updating task-related information during implementation. It has been tested and evaluated in simulation in object transportation, area surveillance, and multi-robot box pushing problem. (Wawerla and Vaughan, 2010) presented two task allocation strategies for a multi-robot transportation system. One is based on a centralized planner that uses domain knowledge to solve the assignment 
problem in linear time. The other enables individual robots to make individual task allocation decisions using only locally obtainable information and single value communication. (Yan et al., 2011) developed a lightweight and robust decentralized approach based on trade rules for coordinated multi-robot exploration (trade-based approach). This approach is designed to simulate the relationship between buyers and sellers in a business system, to achieve dynamic task allocation by using a mechanism of unsolicited bid. A comparison between market-based, auctionbased and trade-based approaches is given in Table 1.

Eventually, the task allocation for heterogeneous and homogeneous systems may be different. In heterogeneous systems, task allocation may be determined by individual capabilities. (Parker, 1994b) introduced the concept of task coverage, which measures the ability of a given team member to achieve a given task. This parameter can be used as an index to organize a robot team to perform a mission from the available pool of heterogeneous robots. Task coverage reaches the maximum value in homogeneous teams, and decreases as teams become more heterogeneous. But in homogeneous systems, agents may need to differentiate into different roles at design time or dynamically at run time (Yan et al., 2011).

\section{CONCLUSION}

In this paper, we have briefly discussed the existing works on the problem of multi-robot task planning (MRTP). Two important aspects, multi-robot task decomposition (MRTD) and multi-robot task allocation (MRTA), were identified. Three online MRTA methods based on contract net protocol, market-based, auction-based and trade-based, were compared with detailed results. Because there is no common benchmarking platform and planning problems, thus the purpose of the comparison is not to point out which method is the best, but give an overview to improve our understanding. Different strategies might be suited to different planning scenarios.

We believe that the following are some promising avenues for future research:

- The task decomposition can directly affect the efficiency of task allocation. A more complete theory should be achieved.

- Environments where robots work in are usually dynamic. Due to the unpredictability and uncertainty of the environment, the revenue and cost functions for task allocation will be difficult to define. For the purpose of maximizing system per- formance, robust definitions and metrics for various scenarios should be developed.

- The motion of the robot is also an important factor influencing task achievement. A future challenge is to combine both task and motion planning reasonably, in order to make the multi-robot systems can be situated to more complex tasks in more complex environments.

\section{REFERENCES}

Botelho, S. C. and Alami, R. (1999). M+: A scheme for multi-robot cooperation through negotiated task allocation and achievement. In Proceedings of the 1999 IEEE International Conference on Robotics and Automation (ICRA'99), pages 1234-1239, Detroit, MI, USA.

Chaimowicz, L., Campos, M. F. M., and Kumar, V. (2002). Dynamic role assignment for cooperative robots. In Proceedings of the 2002 IEEE International Conference on Robotics and $\mathrm{Au}$ tomation (ICRA'02), pages 293-298, Washington, DC, USA.

Chen, J., Yang, Y., and Wei, L. (2010). Research on the approach of task decomposition in soccer robot system. In Proceedings of the 1st International Conference on Digital Manufacturing and Automation (ICDMA'10), pages 284-289, Changsha, China.

Dahl, T. S., Matarić, M. J., and Sukhatme, G. S. (2009). Multi-robot task allocation through vacancy chain scheduling. Robotics and Autonomous Systems, 57(6-7):674-687.

Dias, M. B., Zlot, R., Kalra, N., and Stentz, A. (2005). Market-based multirobot coordination: A survey and analysis. Technical Report CMU-RI-TR-0513, Carnegie Mellon University, Pittsburgh, PA, USA.

Gerkey, B. P. and Matarić, M. J. (2002). Sold!: Auction methods for multirobot coordination. IEEE Transactions on Robotics and Automation, 18(5):758-768.

Gerkey, B. P. and Matarić, M. J. (2004). A formal analysis and taxonomy of task allocation in multi-robot systems. The International Journal of Robotics Research, 23(9):939-954.

Hanna, H. (2005). Decentralized approach for multirobot task allocation problem with uncertain task execution. In Proceedings of the 2005 IEEE/RSJ International Conference on Intelligent Robots and Systems (IROS'05), pages 535-540, Alberta, Canada. 
Table 1: A Comparison of Three CNP-based Online Multi-robot Task Allocation Methods

\begin{tabular}{|l||c|c|c|c|}
\hline Name & $\begin{array}{c}\text { Negotiation } \\
\text { (Communication) } \\
\text { model }\end{array}$ & $\begin{array}{c}\text { Task } \\
\text { allocation } \\
\text { algorithm }\end{array}$ & $\begin{array}{c}\text { Task allocation ability } \\
\text { per iteration } \\
\text { (trading round) }\end{array}$ \\
\hline \hline market-based & publish/subscribe & greedy algorithm (first-price auction) & single task \\
\hline auction-based & publish/subscribe & greedy algorithm (first-price auction) & \multicolumn{2}{c|}{ single task } \\
\hline trade-based & apply/allocate & greedy algorithm (first-price auction) & multiple tasks \\
\hline \hline Name & $\begin{array}{c}\text { Utility } \\
\text { consideration }\end{array}$ & $\begin{array}{c}\text { Task } \\
\text { reassignment }\end{array}$ & $\begin{array}{c}\text { Communication } \\
\text { complexity }\end{array}$ & $\begin{array}{c}\text { Computation } \\
\text { complexity }\end{array}$ \\
\hline \hline market-based & cost and benefit & allowed & $O(1) /$ bidder, $O(n) /$ auctioneer & $O(n)$ \\
\hline auction-based & cost & not allowed & $O(1) /$ bidder, $O(n) /$ auctioneer & $O(n)$ \\
\hline trade-based & cost & allowed & $O(1) /$ buyer, $O(n) /$ seller & $O(n)$ \\
\hline
\end{tabular}

Michael, N., Zavlanos, M. M., Kumar, V., and Pappas, G. J. (2008). Distributed multi-robot task assignment and formation control. In Proceedings of the 2008 IEEE International Conference on Robotics and Automation (ICRA'08), pages 128-133, Pasadena, CA, USA.

Parker, L. E. (1994a). ALLIANCE: An architecture for fault tolerant, cooperative control of heterogeneous mobile robots. In Proceedings of the 1994 IEEE/RSJ International Conference on Intelligent Robots and Systems (IROS'94), pages 776-783, Munich, Germany.

Parker, L. E. (1994b). Heterogeneous Multi-Robot Cooperation. $\mathrm{PhD}$ thesis, Massachusetts Institute of Technology.

Russell, S. J. and Norvig, P. (2002). Artificial Intelligence: A Modern Approach (2nd Edition). Prentice Hall.

Shiroma, P. M. and Campos, M. F. M. (2009). CoMutaR: A framework for multi-robot coordination and task allocation. In Proceedings of the 2009 IEEE/RSJ International Conference on Intelligent Robots and Systems (IROS'09), pages 4817-4824, St. Louis, MO, USA.

Smith, R. G. (1980). The contract net protocol: Highlevel communication and control in a distributed problem solver. IEEE Transactions on Computers, 29(12):1104-1113.

Stentz, A. (1994). Optimal and efficient path planning for partially-known environments. In Proceedings of the 1994 IEEE International Conference on Robotics and Automation (ICRA'94), pages 3310-3317, San Diego, CA, USA.

Stentz, A. and Dias, M. B. (1999). A free market architecture for coordinating multiple robots. Technical Report CMU-RI-TR-99-42, Carnegie Mellon University, Pittsburgh, PA, USA.

Stone, P. and Veloso, M. (1999). Task decomposition, dynamic role assignment, and low-bandwidth communication for real-time strategic teamwork. Artificial Intelligence, 110(2):241-273.

Tang, F. and Parker, L. E. (2005). Asymtre: Automated synthesis of multi-robot task solutions through software reconfiguration. In Proceedings of the 2005 IEEE International Conference on Robotics and Automation (ICRA'05), pages 1501-1508, Barcelona, Spain.

Wawerla, J. and Vaughan, R. T. (2010). A fast and frugal method for team-task allocation in a multi-robot transportation system. In Proceedings of the 2010 IEEE International Conference on Robotics and Automation (ICRA'10), pages 1432-1437, Anchorage, AK, USA.

Werger, B. B. and Matarić, M. J. (2000). Broadcast of local eligibility for multi-target observation. In Proceedings of the 5th International Symposium on Distributed Autonomous Robotic Systems (DARS'O0), pages 347-356, Knoxville, TN, USA.

Yan, Z., Jouandeau, N., and Ali Cherif, A. (2011). Multi-robot decentralized exploration using a trade-based approach. In Proceedings of the 8th International Conference on Informatics in Control, Automation and Robotics (ICINCO'11), pages 99-105, Noordwijkerhout, The Netherlands.

Zlot, R. and Stentz, A. (2005). Complex task allocation for multiple robots. In Proceedings of the 2005 IEEE International Conference on Robotics and Automation (ICRA'05), pages 1515-1522, Barcelona, Spain.

Zlot, R., Stentz, A. T., Dias, M. B., and Thayer, S. (2002). Multi-robot exploration controlled by a market economy. In Proceedings of the 2002 IEEE International Conference on Robotics and Automation (ICRA'02), pages 3016-2023, Washington, DC, USA. 